\title{
Iron: how much is too much?
}

\author{
Aidan McElduff ${ }^{1}$ \\ Received: 21 November 2016 / Accepted: 23 November 2016/Published online: 9 December 2016 \\ (C) Springer-Verlag Berlin Heidelberg 2016
}

\begin{abstract}
Gross iron overload has long been known to result in diabetes mellitus. However, it is now thought that milder levels of iron overload, possibly within the normal range, also increase the risk of diabetes mellitus. The article by Rawal et al in this issue (Diabetologia doi: 10.1007/s00125-016-4149-3) provides further support for the relationship between mild degrees of iron overload and the risk of gestational diabetes. The purpose of this commentary is to briefly discuss the background of this relationship and the implications it may have for routine pregnancy care.
\end{abstract}

Keywords Diabetes $\cdot$ Diabetes risk $\cdot$ Gestational diabetes · Hyperferritinaemia $\cdot$ Iron overload $\cdot$ Pregnant

\begin{abstract}
Abbreviations
GDM Gestational diabetes mellitus

sTfR Soluble transferrin receptor
\end{abstract}

\section{Introduction}

Iron is an essential element in normal human physiology despite the fact that the free molecule is highly toxic. Iron readily oxidises or reduces and this property can help create powerful oxidant species. There are complex regulatory mechanisms to control iron metabolism and prevent iron toxicity [1]. However, despite these protective mechanisms,

Aidan McElduff

aidan.mcelduff@sydney.edu.au

1 The Discipline of Medicine, Sydney Medical School, The University of Sydney, Sydney, NSW 2006, Australia it is well known that gross iron overload is toxic and results in a number of pathophysiological conditions including diabetes mellitus.

\section{The aetiology of diabetes and iron overload}

The aetiology of diabetes in states of iron overload is complex [2-4]; insulin deficiency may be the primary problem, as I was taught as a medical student. Insulin resistance also contributes to this process, as does the disruption of almost all the metabolic pathways that involve iron, leading to metabolic inflexibility [3, 4]. However, insulin resistance may well be secondary to other factors, including hyperglycaemia.

It should not be assumed that the mechanisms that account for the increased risk of diabetes with gross iron overload are the same as the risk that has more recently been identified with milder iron excess. Over the last 20 years or so, evidence has grown that more subtle levels of iron overload, or even sufficiency, are associated with diabetes and a causal link has been suggested [5]. Epidemiological studies have demonstrated a relationship between measures of iron stores (predominantly ferritin) and the risk of developing typical type 2 diabetes [6] or gestational diabetes [7], and of having these conditions [2]. However, since diabetes is associated with inflammation, which causes elevations in ferritin levels, the causal relationship between iron stores measured by ferritin and diabetes has been questioned.

\section{Evidence for the impact of iron status on diabetes risk}

Evidence used to support a causal relationship between iron status and diabetes includes the observation that the risk of 
developing type 2 diabetes is approximately three times greater following an increment of $5 \mathrm{mg} /$ day of dietary haem iron [7-9]. Non-haem iron intake, as well as supplemental iron, seems not to be associated with type 2 diabetes [7-9]. However, this evidence should be judged cautiously given the reduced absorption of non-haem iron compared with haem iron and the problems of compliance with supplements. Such a conclusion regarding iron supplements may be important, however, as it may reduce concerns about iron therapy in pregnancy.

Stronger evidence for the impact of iron status on diabetes, although based on imperfect data, is the observation that venesection may protect against type 2 diabetes [10] or improve glycaemic control in healthy individuals [11] and in people with impaired glucose tolerance [12]. Venesection also improves insulin sensitivity and glycaemic control in type 2 diabetes, particularly in participants with high ferritin values $[13,14]$. These observations need to be confirmed in a large randomised controlled trial; if the benefits of venesection on diabetes and diabetic risk were to be confirmed, it would change clinical practice.

\section{Surrogate markers for iron storage: which one should be used to assess risk and when?}

In this issue of Diabetologia, Rawal et al [15] describe a relatively small case-control study that provides further support for the hypothesis that a mild degree of excess iron storage increases the risk of developing diabetes, in this case gestational diabetes mellitus (GDM).

The identification of excess iron stores relied on indirect measurements, namely of ferritin, hepcidin and soluble transferrin receptor (sTfR), and persisted despite correction for several confounders, including $\mathrm{C}$-reactive protein (CRP) as an indicator of inflammation, and several obvious risk factors for GDM. There was no relationship between GDM risk and sTfR, possibly because this variable is reflective of iron deficiency (sTfR levels increase as iron deficiency worsens) and the relationship between the risk of diabetes and iron stores does not persist at lower levels of iron storage. Rawal and colleagues [15] also demonstrated that the relationship between GDM risk and iron status was not uniform when defined by ferritin and hepcidin; the positive association between hepcidin and the risk of GDM was only present in the second trimester, whereas with ferritin this relationship occurred in the first trimester (Table 1). Hepcidin and ferritin levels are both increased with inflammation, but hepcidin production is otherwise thought to increase only with increased iron stores.

If the hypothesis that abundant iron stores increase the risk of diabetes is correct, defining the precise relationship between iron load and the risk of hyperglycaemia is important. For example, does the increased risk of GDM commence with levels of iron stores within the 'high end' of the normal range or only with increased iron storage levels above the reference range? Defining the threshold for risk (if a threshold exists) and when a 'normal' result may not necessarily signify the absence of risk will be important for clinical practice. Additionally, defining the optimal marker for elevated iron storage will also be necessary to advance clinical practice.

Rawal et al [15] also showed that there was no association between any of the measures of iron stores and GDM risk in the later stages of pregnancy. This finding may reflect the changes that occur in iron stores in late pregnancy. However, it may also be a result of the decreased number of cases and controls at later stages of the study compared with at the first trimester. Nonetheless, findings that contribute to our understanding of the mechanisms underlying any relationship between excess iron stores and the risk of developing diabetes, and the pathophysiology of diabetes in general, are particularly important. It is also important for us to understand the relationship between surrogate measures of iron stores and diabetes, particularly if inflammation impacts on levels of these variables, causing them to be elevated. The aetiological mechanisms underlying the relationship between measures of iron stores and the risk of hyperglycaemia are likely to be multifactorial and are predicted to play only a modest role in the development of subsequent hyperglycaemia. This is in keeping with the current concept of the aetiology of type 2 diabetes, which is the sum of many minor abnormalities.

Table 1 Potential relationship between iron status and the risk of diabetes

\begin{tabular}{llll}
\hline Iron status & Diabetes risk & GDM risk & Marker of GDM risk \\
\hline $\begin{array}{l}\text { Depleted } \\
\text { Sufficient }\end{array}$ & Not increased & Not increased & sTfR \\
Oossibly increased at high 'normal' levels & (as reflected by ferritin levels) & Not clear & \\
& Increased & Increased & Ferritin (first and second trimesters) \\
& & & Hepcidin (second trimester) \\
\hline
\end{tabular}

Data for GDM risk and markers of risk are taken from [15] 


\section{Implications for clinical practice}

Do these findings have any implications for clinical practice at this time? That may depend on where you practice. Rawal et al [15] note that guidelines for iron therapy in pregnancy vary. In areas of the world in which iron deficiency in pregnancy is common and resources are limited, the WHO and Centers for Disease Control and Prevention recommendations for routine iron supplementation in pregnancy seem appropriate. In areas where testing for iron deficiency in pregnancy is routine, iron supplementation should be used only as required.

Further research in this area is required; intervention studies in individuals with elevated ferritin levels may help to confirm the benefits of reducing ferritin levels with regard to diabetes prevention or improvements in glycaemic control in those with varying degrees of glucose intolerance. This research would not address the underlying mechanisms involved in the increase in GDM risk with excess iron storage but would change clinical practice and influence pre-pregnancy planning.

\section{Conclusions}

In an ideal world, confirmation of the findings reported by Rawal et al [15] in a larger, prospective population study with multivariate analysis correcting for all known confounders would be very useful and could influence the approach to iron therapy in pregnancy. In the meantime, the status quo seems appropriate while awaiting further studies, particularly on the potential benefits of venesection.

Duality of interest The author declares that there is no duality of interest associated with this manuscript.

Contribution statement The author was the sole contributor to this paper.

\section{References}

1. Waldvogel-Abramowski S, Waeber G, Gassner C et al (2014) Physiology of iron metabolism. Transfus Med Hemother 41:213-221

2. Swaminathan S, Fonseca VA, Alam MG, Shah SV (2007) The role of iron in diabetes and its complications. Diabetes Care 30:1926-1933

3. Simcox JA, McClain DA (2013) Iron and diabetes risk. Cell Metab 17:329-341

4. Huang J, Jones D, Luo B et al (2011) Iron overload and diabetes risk: a shift from glucose to fatty acid oxidation and increased hepatic glucose production in a mouse model of hereditary hemochromatosis. Diabetes 60:80-87

5. Kunutsor SK, Apekey TA, Walley J, Kain K (2013) Ferritin levels and risk of type 2 diabetes mellitus: an updated systematic review and meta-analysis of prospective evidence. Diabetes Metab Res Rev 29:308-318

6. Forouhi NG, Harding AH, Allison M et al (2007) Elevated serum ferritin levels predict new-onset type 2 diabetes: results from the EPIC- Norfolk prospective study. Diabetologia 50:949-956

7. Khambalia AZ, Aimone A, Nagubandi P et al (2015) High maternal iron status, dietary iron intake and iron supplement use in pregnancy and risk of gestational diabetes mellitus: a prospective study and systematic review. Diabet Med 33:1211-1221

8. Waldvogel-Abramovski S, Waeber G, Gassner C, Buser A, Frey BM, Favrat B, Tissot J-D (2014) Iron and transfusion medicine. Blood Rev 27:289-295

9. Bao W, Rong Y, Rong S, Liu L (2012) Dietary iron intake, body iron stores, and the risk of type 2 diabetes: a systematic review and meta-analysis. BMC Med 10:119-132

10. Ascherio A, Rimm EB, Giovannucci E, Willett WC, Stampfer MJ (2001) Blood donations and risk of coronary heart disease in men. Circulation 103:52-57

11. Facchini FS (1998) Effect of phlebotomy on plasma glucose and insulin concentrations. Diabetes Care 21:2190

12. Houschyar KS, Ludtke R, Dobos et al (2012) Effects of phlebotomyinduced reduction of body iron stores on metabolic syndrome: results from a randomized clinical trial. BMC Med 10:54-62

13. Fernandez-Real JM, Penarroja G, Castro et al (2002) Blood letting in high-ferritin type 2 diabetes: effects on insulin sensitivity and beta-cell function. Diabetes 51:1000-1004

14. Wilson MS, Thompson EW (2016) Venesection is an effective nonpharmacological treatment for patients with high serum ferritin type 2 diabetes. J Clin Transl Endocrinol: Case Rep doi:10.1016/j. jecr.2016.10.002

15. Rawal S, Hinkle SN, Bao W et al (2016) A longitudinal study of iron status during pregnancy and the risk of gestational diabetes: findings from a prospective, multiracial cohort. Diabetologia. doi:10.1007/s00125-016-4149-3 